STABILITY IN PROBABILITY

BANACH CENTER PUBLICATIONS, VOLUME 90

INSTITUTE OF MATHEMATICS

POLISH ACADEMY OF SCIENCES

WARSZAWA 2010

\title{
GENERALIZED TEMPERED STABLE PROCESSES
}

\author{
JAN ROSIŃSKI \\ Department of Mathematics, University of Tennessee \\ 1534 Cumberland Ave., Knoxville, TN 37996, USA \\ E-mail: rosinski@math.utk.edu \\ JENNIFER L. SINCLAIR \\ School of Science and Technology, Georgia Gwinnett College \\ 1000 University Center Lane, Lawrenceville, GA 30043, USA \\ E-mail: jsincla1@ggc.edu
}

\begin{abstract}
This work introduces the class of generalized tempered stable processes which encompass variations on tempered stable processes that have been introduced in the field, including "modified tempered stable processes", "layered stable processes", and "Lamperti stable processes". Short and long time behavior of GTS Lévy processes is characterized and the absolute continuity of GTS processes with respect to the underlying stable processes is established. Series representations of GTS Lévy processes are derived. Such representations can be used for simulation and illustration of GTS processes as well as for their theoretical study.
\end{abstract}

1. Introduction. Tempered stable distributions have been introduced as a modification of stable laws to model systems exhibiting local spatiotemporal fractality and aggregational Gaussianity. It has been known that the velocity change in a turbulent flow is extremely chaotic (fractal) in a short period of time but over long periods of time it can be modeled by Gaussian distributions. Physicists Mantegna and Stanley [10] proposed stable distributions with truncated Lévy measure to model such phenomena. The papers of Novikov [11 and Koponen 9] replaced truncation by exponential tilting and put the ideas of [10] on a solid mathematical ground. Presently tempered stable models are used in several areas of physics, including turbulence, plasma physics, solar winds, and their popularity is still growing.

2010 Mathematics Subject Classification: 60E07, 60F05, 60G51, 60G52.

Key words and phrases: tempered stable distributions and processes, stable processes, Lévy processes, series representations of Lévy processes.

The paper is in final form and no version of it will be published elsewhere. 
Analogous behavior to turbulent velocity was observed in the study of changes of certain stocks on financial markets. To model such phenomena, Carr, Geman, Madan, and Yor 4] introduced exponentially tempered stable processes (the CGMY model). Barndorff-Nielsen and Shephard developed non-Gaussian Ornstein-Uhlenbeck-based models applying, among others, exponentially tempered stable processes. At present, there is a substantial literature in financial mathematics devoted to the CGMY and BarndorffNielsen and Shephard models.

Exponentially tempered stable processes have their natural mathematical limitations. For example, they are not closed under superpositions of independent terms, and the tails are always decreasing exponentially fast. To gain a bigger flexibility but yet to retain nice features of exponentially tempered stable processes, Rosiński [13 introduced tempered stable distributions, where tempering is due to a radial completely monotone function. He also found special series representations of such distributions and processes based on their intrinsic characteristics. Simulation methods of such processes based on such series representations and Gaussian approximation of small jumps were investigated by Cohen and Rosiński [5. Terdik and Woyczyński [15] studied in detail tempered stable distribution under several parametric classes of tempering functions.

Several authors introduced different modifications of tempered stable distributions and processes. Houdré and Kawai [6] introduced layered stable processes, Kim, Rachev, Chung, and Bianchi [8] introduced modified stable processes, and Caballero, Pardo, and Pérez [3] introduced Lamperti stable processes. The purpose of this work is to identify the essential feature of all four classes of tempered-like Lévy processes that enables us to prove stable behavior in short time scales, Gaussian in long time scales, and absolute continuity with respect to underlying stable processes. The resulting distributions or processes we call generalized tempered stable (GTS) distributions or processes, respectively.

In Section 2 we give the definition of a GTS distribution and discuss examples. Short and long time behavior of GTS Lévy processes is proved in Section 3. In Section 4 we prove the absolute continuity of GTS processes with respect to the underlying stable process. Series representation of GTS Lévy processes are considered in Section 5. We examine two methods of generating series representations in the context of GTS processes: the LePage Method and the Rejection Method. We establish general forms of representations of GTS processes and compute examples. Such series representations can be used for simulation of GTS processes.

\section{Generalized tempered stable distributions}

Definition 2.1. An infinitely divisible distribution $\mu$ on $\mathbb{R}^{d}$ is said to be generalized tempered stable (GTS) if $\mu$ has no Gaussian part and the Lévy measure $\nu$ of $\mu$ can be represented as

$$
\nu(B)=\int_{S^{d-1}} \int_{0}^{\infty} 1_{B}(r \xi) q(r, \xi) r^{-\alpha-1} d r \sigma(d \xi), \quad B \in \mathcal{B}\left(\mathbb{R}^{d}\right),
$$


where $\alpha \in(0,2), \sigma$ is a finite measure on $S^{d-1}$, and $q:(0, \infty) \times S^{d-1} \mapsto \mathbb{R}_{+}$is a measurable function such that for some nonnegative function $g \in L^{1}\left(S^{d-1}, \sigma\right)$

$$
\lim _{r \rightarrow 0}\|q(r, \cdot)-g(\cdot)\|_{L^{1}\left(S^{d-1}, \sigma\right)}=0 .
$$

We write $\nu^{G T S}$ to signify that this is the Lévy measure of a GTS distribution as in (1).

The class of GTS distributions contains various "tempered-like" distributions that were earlier considered for their own merit. We will list some of them.

ExAmple 2.2 (Tempered Stable (TS) Distributions, [13]). These are infinitely divisible distributions on $\mathbb{R}^{d}$ with no Gaussian parts and Lévy measures of the form (1) such that for each $\xi \in S^{d-1}$, the function $q(\cdot, \xi)$ is a completely monotone function on $(0, \infty)$ satisfying $q(\infty, \xi)=0$, and $q(0+, \xi)=1$. (We refer here to proper tempered stable distributions, according to the terminology of [13.)

The complete monotonicity means that $(-1)^{n} \frac{\partial^{n}}{\partial r^{n}} q(r, \xi)>0$ for all $r>0, \xi \in S^{d-1}$, and $n=0,1,2, \ldots$ In particular, $q(\cdot, \xi)$ is strictly decreasing, convex, and since $q(0+, \xi)=$ 1 , it is bounded by 1 . Thus condition (2) holds with $g(\xi)=1$. A function $q(r, \xi)=e^{-\lambda r}$ is a standard example of the $q$-function for a tempered stable distribution. For classes of more interesting examples of tempering see [15].

ExAmple 2.3 (Layered Stable Distributions, 6]). These are infinitely divisible distributions on $\mathbb{R}^{d}$ with no Gaussian parts and Lévy measures of the form $(1)$ with $q(r, \cdot)$ being locally integrable and such that

$$
q(r, \xi) \sim c_{1}(\xi) \quad \text { as } r \rightarrow 0
$$

and

$$
r^{\beta-\alpha} q(r, \xi) \sim c_{2}(\xi) \quad \text { as } r \rightarrow \infty
$$

for $\sigma$-almost every $\xi \in S^{d-1}$. Here $c_{1}$ and $c_{2}$ are positive functions in $L^{1}\left(S^{d-1}, \sigma\right), \alpha \in$ $(0,2)$ and $\beta \in(0, \infty)$.

Layered stable distributions inspired our way to introduce GTS distributions. However, condition (3) is not sufficient for the stable behavior of a layered stable process in a short time, contrary to the claim made in Theorem 3.1 of [6], as a simple counterexample can be constructed. For that reason we introduce (2). We do not require (4) as it is not satisfied by other examples. A layered stable distribution becomes a GTS distribution with $g=c_{1}$ when we add the convergence in $L^{1}\left(S^{d-1}, \sigma\right)$ to $(3)$.

ExAmple 2.4 (Modified Tempered Stable (MTS) Distributions, [8]). These are infinitely divisible distributions on $\mathbb{R}$ with no Gaussian parts and Lévy measures of the form (1) with

$$
q(r, \xi)= \begin{cases}2^{\frac{1-\alpha}{2}}\left(\Gamma\left(\frac{\alpha+1}{2}\right)\right)^{-1}\left(\lambda_{+} r\right)^{\frac{\alpha+1}{2}} K_{\frac{\alpha+1}{2}}\left(\lambda_{+} r\right) & \text { if } \xi=1, \\ 2^{\frac{1-\alpha}{2}}\left(\Gamma\left(\frac{\alpha+1}{2}\right)\right)^{-1}\left(\lambda_{-} r\right)^{\frac{\alpha+1}{2}} K_{\frac{\alpha+1}{2}}\left(\lambda_{-} r\right) & \text { if } \xi=-1,\end{cases}
$$

where $\lambda_{+}, \lambda_{-}>0, \alpha \in(0,2)$, and $K_{p}$ is the modified Bessel function of the second kind 
with an integral representation given by

$$
K_{p}(x)=\frac{1}{2}\left(\frac{x}{2}\right)^{p} \int_{0}^{\infty} e^{-t-\frac{x^{2}}{4 t}} t^{-p-1} d t .
$$

Since for any $p>0$, the function $x \mapsto x^{p} K_{p}(x)$ is decreasing with $\lim _{x \rightarrow 0} x^{p} K_{p}(x)=$ $2^{p-1} \Gamma(p)$ and $\lim _{x \rightarrow \infty} x^{1 / 2} e^{x} K_{p}(x)=(\pi / 2)^{1 / 2}$, we get that for each $\xi= \pm 1$ the radial tempering function $r \mapsto q(r, \xi)$ is decreasing with

$$
q(r, \xi) \sim 1 \text { as } r \downarrow 0 \quad \text { and } \quad q(r, \xi) \sim\left(\frac{\pi}{2}\right)^{1 / 2}\left(\lambda_{ \pm} r\right)^{\alpha / 2} e^{-\lambda_{ \pm} r} \text { as } r \uparrow \infty .
$$

Thus MTS distributions belong to the class of GTS distributions with $g(\xi)=1$ in (2). We notice that an MTS distribution is not a special case of a TS distribution because the second derivative of $q(r, \xi)$ with respect to $r$ fails to be positive for all $r>0$. This is not a layered stable distribution either, because $q(\cdot, \xi)$ decays at infinity faster than any power function.

Example 2.5 (Lamperti Stable Distributions, [3]). These are infinitely divisible distributions on $\mathbb{R}^{d}$ with no Gaussian parts and Lévy measures of the form (1) with

$$
q(r, \xi)=\frac{e^{r f(\xi)} r^{\alpha+1}}{\left(e^{r}-1\right)^{\alpha+1}},
$$

where $\alpha \in(0,2)$ and $f: S^{d-1} \rightarrow \mathbb{R}$ is such that $\beta:=\sup _{\xi \in S^{d-1}} f(\xi)<\alpha+1$. We have

$$
q(r, \xi) \sim 1 \text { as } r \downarrow 0 \quad \text { and } \quad q(r, \xi) \sim r^{\alpha+1} e^{-(\alpha+1-f(\xi)) r} \text { as } r \uparrow \infty .
$$

It is easy to see that there is a finite constant $C(\alpha, \beta)$ such that $q(r, \xi) \leq C(\alpha, \beta)$. Thus Lamperti stable distributions belong to the class of GTS distributions with $g(\xi)=1$ in (2). However, as noted in [3], Lamperti stable distributions are not tempered stable nor layered stable. This is because the function $r \mapsto q(r, \xi)$ fails to be completely monotone and decays at infinity faster than any power function.

The following is an immediate consequence of 22 . We state it for a convenient reference. There exists $\delta \in(0,1]$ such that

$$
\int_{S^{d-1}} q(r, \xi) \sigma(d \xi) \leq \int_{S^{d-1}} g(\xi) \sigma(d \xi)+1=: M_{1} \quad \forall r \in(0, \delta] .
$$

Lemma 2.6. Suppose $\alpha \in(0,1)$. Then

$$
\int_{\{\|x\| \leq 1\}}\|x\| \nu^{G T S}(d x)<\infty .
$$

Proof. Using (6) we get

$$
\begin{aligned}
\int_{\{\|x\| \leq 1\}}\|x\| \nu^{G T S}(d x) & \leq \int_{\{\|x\| \leq \delta\}}\|x\| \nu^{G T S}(d x)+\nu^{G T S}(\{\|x\|>\delta\}) \\
& =\int_{S^{d-1}} \int_{0}^{\delta} r^{-\alpha} q(r, \xi) d r \sigma(d \xi)+\nu^{G T S}(\{\|x\|>\delta\}) \\
& \leq M_{1} \int_{0}^{\delta} r^{-\alpha} d r+\nu^{G T S}(\{\|x\|>\delta\})<\infty .
\end{aligned}
$$


Consequently, the characteristic function $\widehat{\mu}$ of a GTS distribution can be written as $\widehat{\mu}(y)=\exp \left(C_{\mu}(y)\right)$, where the cumulant function $C_{\mu}(y), y \in \mathbb{R}^{d}$, is given by

$$
C_{\mu}(y)= \begin{cases}\int_{\mathbb{R}^{d}}\left(e^{i\langle y, x\rangle}-1\right) \nu^{G T S}(d x)+i\langle y, b\rangle, & \alpha \in(0,1), \\ \int_{\mathbb{R}^{d}}\left(e^{i\langle y, x\rangle}-1-i\langle y, x\rangle \mathbf{1}_{\{\|x\| \leq 1\}}(x)\right) \nu^{G T S}(d x)+i\langle y, b\rangle, & \alpha \in[1,2) .\end{cases}
$$

We will also write $\mu=\mu^{G T S}$ to signify that $\mu$ is a GTS distribution.

Definition 2.7. Given a Lévy measure $\nu^{G T S}$, as in Definition 2.1, define the associated $\alpha$-stable Lévy measure by

$$
\nu^{\alpha}(B)=\int_{S^{d-1}} \int_{0}^{\infty} \mathbf{1}_{B}(r \xi) r^{-\alpha-1} d r g(\xi) \sigma(d \xi), \quad B \in \mathcal{B}\left(\mathbb{R}^{d}\right) .
$$

If $\mu$ is a GTS distribution with the cumulant function as in 77 , then the distribution $\mu^{\alpha}$ with the cumulant function

$$
C_{\mu^{\alpha}}(y)= \begin{cases}\int_{\mathbb{R}^{d}}\left(e^{i\langle y, x\rangle}-1\right) \nu^{\alpha}(d x)+i\langle y, b\rangle, & \alpha \in(0,1), \\ \int_{\mathbb{R}^{d}}\left(e^{i\langle y, x\rangle}-1-i\langle y, x\rangle \mathbf{1}_{\{\|x\| \leq 1\}}(x)\right) \nu^{\alpha}(d x)+i\langle y, b\rangle, & \alpha \in[1,2),\end{cases}
$$

will be called the associated $\alpha$-stable distribution. In short, the associated $\alpha$-stable distribution $\mu^{\alpha}$ has the spectral measure $g(\xi) \sigma(d \xi)$ and drifts analogous to $\mu^{G T S}$.

3. Short and long time behavior of GTS Lévy processes. Let $\left\{X_{t}^{G T S}\right\}_{t \geq 0}$ be a Lévy process in $\mathbb{R}^{d}$ determined by a GTS distribution $\mu$ of $X_{1}^{G T S}$ (in short, a GTS Lévy process). A Lévy process $\left\{X_{t}^{\alpha}\right\}_{t \geq 0}$ such that $X_{1}^{\alpha}$ has an $\alpha$-stable distribution associated to $\mathcal{L}\left(X_{1}^{G T S}\right)$ is called the associated $\alpha$-stable Lévy process.

The following theorem extends similar results obtained for special cases of GTS Lévy processes. It shows that in a short time a GTS process behaves like a stable one but in a long time (when $q$ decays appropriately) it converges to a Gaussian process.

The convergence in distribution " $\stackrel{d}{\rightarrow}$ " of processes is understood in $D\left([0, \infty), \mathbb{R}^{d}\right)$, in the Skorohod topology.

THEOREM 3.1. Consider a Lévy process $\left\{X_{t}^{G T S}\right\}_{t \geq 0}$ such that $X_{1}$ has a GTS distribution $\mu$ with the cumulant function as in (7) with $b=0$. Put

$$
a_{h}= \begin{cases}0, & \alpha \in(0,1), \\ -\int_{\left\{h^{1 / \alpha}<\|x\| \leq 1\right\}} x \nu^{G T S}(d x), & \alpha \in[1,2) .\end{cases}
$$

Case(i): (Short time) If $h \rightarrow 0$, then

$$
\left\{h^{-1 / \alpha}\left[X_{h t}^{G T S}-h t a_{h}\right]\right\}_{t \geq 0} \stackrel{d}{\rightarrow}\left\{X_{t}^{\alpha}\right\}_{t \geq 0}
$$

where $\left\{X_{t}^{\alpha}\right\}_{t \geq 0}$ is the associated $\alpha$-stable Lévy process.

Case(ii): (Long time) Assume additionally that

$$
\int_{\{\|x\|>1\}}\|x\|^{2} \nu^{G T S}(d x)<\infty
$$


and define

$$
b_{h}= \begin{cases}\int_{\mathbb{R}^{d}} x \nu^{G T S}(d x), & \alpha \in(0,1), \\ \int_{\{\|x\|>1\}} x \nu^{G T S}(d x), & \alpha \in[1,2) .\end{cases}
$$

Then, as $h \rightarrow \infty$,

$$
\left\{h^{-1 / 2}\left[X_{h t}^{G T S}-h t b_{h}\right]\right\}_{t \geq 0} \stackrel{d}{\rightarrow}\left\{B_{t}\right\}_{t \geq 0}
$$

where $\left\{B_{t}\right\}_{t \geq 0}$ is a centered Brownian motion with covariance matrix

$$
\Sigma=\int_{\mathbb{R}^{d}} x x^{\top} \nu^{G T S}(d x)
$$

Proof. Case (i): Short time $(h \rightarrow 0)$. Since for each $h>0, Y_{t}^{h}:=h^{-1 / \alpha}\left[X_{h t}^{G T S}-h t a_{h}\right]$ is a Lévy process, by a theorem of Skorohod (see [7, Theorem 15.17]) we only need to check the convergence at time 1 . Therefore, we need to show that

$$
Y_{1}^{h} \stackrel{d}{\rightarrow} X_{1}^{\alpha} \quad \text { as } h \rightarrow 0 .
$$

In view of (7), the cumulant $C_{Y_{1}^{h}}$ of $Y_{1}^{h}$ is given by

$$
\begin{aligned}
& C_{Y_{1}^{h}}(y)=h C_{\mu}\left(h^{-1 / \alpha} y\right)-i h^{1-1 / \alpha}\left\langle y, a_{h}\right\rangle \\
& = \begin{cases}h \int_{\mathbb{R}^{d}}\left(e^{i\left\langle y, h^{-1 / \alpha} x\right\rangle}-1\right) \nu^{G T S}(d x), & \alpha \in(0,1), \\
h \int_{\mathbb{R}^{d}}\left(e^{i\left\langle y, h^{-1 / \alpha} x\right\rangle}-1-i\left\langle y, h^{-1 / \alpha} x\right\rangle \mathbf{1}_{\{\|x\| \leq 1\}}\left(h^{-1 / \alpha} x\right)\right) \nu^{G T S}(d x), & \alpha \in[1,2) .\end{cases}
\end{aligned}
$$

This can be written as

$$
C_{Y_{1}^{h}}(y)= \begin{cases}\int_{\mathbb{R}^{d}}\left(e^{i\langle y, x\rangle}-1\right) \lambda^{h}(d x), & \alpha \in(0,1), \\ \int_{\mathbb{R}^{d}}\left(e^{i\langle y, x\rangle}-1-i\langle y, x\rangle \mathbf{1}_{\{\|x\| \leq 1\}}(x)\right) \lambda^{h}(d x), & \alpha \in[1,2),\end{cases}
$$

where $\lambda^{h}(B):=h \nu^{G T S}\left(h^{1 / \alpha} B\right), B \in \mathcal{B}\left(\mathbb{R}^{d}\right)$ is the Lévy measure of $Y_{1}^{h}$.

We will prove (14) using [7, Theorem 15.14]. To this end we need to verify the following conditions: as $h \rightarrow 0$,

(a) $\lambda^{h}$ converges vaguely to $\nu^{\alpha}$ on $\overline{\mathbb{R}^{d}} \backslash\{0\}$,

(b) $\int_{\{\|x\| \leq 1\}} x x^{\top} \lambda^{h}(d x) \rightarrow \int_{\{\|x\| \leq 1\}} x x^{\top} \nu^{\alpha}(d x)$,

(c) $\int_{\{\|x\| \leq 1\}} x \lambda^{h}(d x) \rightarrow \int_{\{\|x\| \leq 1\}} x \nu^{\alpha}(d x)$ when $\alpha \in(0,1)$.

Here $\overline{\mathbb{R}^{d}}$ denotes the one-point compactification of $\mathbb{R}^{d}$. To prove (a) we need to show that for any bounded continuous function $f: \mathbb{R}^{d} \rightarrow \mathbb{R}$, vanishing in a neighborhood of the origin and such that $\lim _{\|x\| \rightarrow \infty} f(x)$ exists, we have

$$
\int_{\mathbb{R}^{d}} f(x) \lambda^{h}(d x) \rightarrow \int_{\mathbb{R}^{d}} f(x) \nu^{\alpha}(d x) \quad \text { as } h \rightarrow 0 .
$$


Let $C=\sup _{\mathbb{R}^{d}}|f(x)|$ and let $r_{0} \in(0,1]$ be such that $f(x)=0$ whenever $\|x\| \leq r_{0}$. Then

$$
\begin{aligned}
\int_{\mathbb{R}^{d}} f(x) \lambda^{h}(d x) & =h \int_{\mathbb{R}^{d}} f\left(h^{-1 / \alpha} x\right) \nu^{G T S}(d x) \\
& =h \int_{S^{d-1}} \int_{h^{1 / \alpha} r_{0}}^{\infty} f\left(h^{-1 / \alpha} r \xi\right) r^{-\alpha-1} q(r, \xi) d r \sigma(d \xi) \\
& =\int_{S^{d-1}} \int_{r_{0}}^{\infty} f(r \xi) r^{-\alpha-1} q\left(h^{1 / \alpha} r, \xi\right) d r \sigma(d \xi) \\
& =\int_{r_{0}}^{\infty} F_{h}(r) r^{-\alpha-1} d r
\end{aligned}
$$

where

$$
F_{h}(r)=\int_{S^{d-1}} f(r \xi) q\left(h^{1 / \alpha} r, \xi\right) \sigma(d \xi), \quad r \geq r_{0}
$$

By condition 2], for each $r \geq r_{0}$,

$$
F_{h}(r) \rightarrow \int_{S^{d-1}} f(r \xi) g(\xi) \sigma(d \xi), \quad \text { as } h \rightarrow 0,
$$

and also, by (6), $\left|F_{h}(r)\right| \leq C M_{1}$ whenever $h \leq \delta^{\alpha} r_{0}^{-\alpha}$. Therefore,

$$
\lim _{h \rightarrow 0} \int_{\mathbb{R}^{d}} f(x) \lambda^{h}(d x)=\int_{r_{0}}^{\infty} \int_{S^{d-1}} f(r \xi) g(\xi) \sigma(d \xi) r^{-\alpha-1} d r=\int_{\mathbb{R}^{d}} f(x) \nu^{\alpha}(d x),
$$

which proves $(16)$ and so (a).

To show (b) we write

$$
\begin{aligned}
\int_{\{\|x\| \leq 1\}} x x^{\top} \lambda^{h}(d x) & =h^{1-2 / \alpha} \int_{\left\{\|x\| \leq h^{1 / \alpha}\right\}} x x^{\top} \nu^{G T S}(d x) \\
& =h^{1-2 / \alpha} \int_{S^{d-1}} \int_{0}^{h^{1 / \alpha}} \xi \xi^{\top} r^{1-\alpha} q(r, \xi) d r \sigma(d \xi) \\
& =\int_{0}^{1} G_{h}(r) r^{1-\alpha} d r,
\end{aligned}
$$

where $G_{h}$ is a matrix valued function given by

$$
G_{h}(r)=\int_{S^{d-1}} \xi \xi^{\top} q\left(h^{1 / \alpha} r, \xi\right) \sigma(d \xi), \quad r \in(0,1] .
$$

$G_{h}$ is uniformly bounded,

$$
\left\|G_{h}(r)\right\| \leq \int_{S^{d-1}} q\left(h^{1 / \alpha} r, \xi\right) \sigma(d \xi) \leq M_{1}
$$

for $h \leq \delta^{\alpha}$ by (6), and $\lim _{h \rightarrow 0} G_{h}(r)=\int_{S^{d-1}} \xi \xi^{\top} g(\xi) \sigma(d \xi)$ by $[2)$. Since $r^{1-\alpha} \mathbf{1}_{(0,1]}(r) d r$ is a finite measure, we obtain by the Dominated Convergence Theorem

$$
\lim _{h \rightarrow 0} \int_{\{\|x\| \leq 1\}} x x^{\top} \lambda^{h}(d x)=\int_{0}^{1} \int_{S^{d-1}} \xi \xi^{\top} g(\xi) \sigma(d \xi) r^{1-\alpha} d r=\int_{\{\|x\| \leq 1\}} x x^{\top} \nu^{\alpha}(d x),
$$

which proves (b). 
To show (c) we notice that, when $\alpha \in(0,1)$,

$$
\begin{aligned}
\int_{\{\|x\| \leq 1\}} x \lambda^{h}(d x) & =h^{1-1 / \alpha} \int_{\left\{\|x\| \leq h^{1 / \alpha}\right\}} x \nu^{G T S}(d x) \\
& =h^{1-1 / \alpha} \int_{S^{d-1}} \int_{0}^{h^{1 / \alpha}} \xi r^{-\alpha} q(r, \xi) d r \sigma(d \xi) \\
& =\int_{0}^{1} H_{h}(r) r^{-\alpha} d r
\end{aligned}
$$

where

$$
H_{h}(r)=\int_{S^{d-1}} \xi q\left(h^{1 / \alpha} r, \xi\right) \sigma(d \xi), \quad r \in(0,1] .
$$

By the same arguments as above we get that $H_{h}$ is uniformly bounded for small $h$ and $\lim _{h \rightarrow 0} H_{h}(r)=\int_{S^{d-1}} \xi g(\xi) \sigma(d \xi)$. Since $r^{-\alpha} \mathbf{1}_{(0,1]}(r) d r$ is a finite measure, we obtain

$$
\lim _{h \rightarrow 0} \int_{\{\|x\| \leq 1\}} x \lambda^{h}(d x)=\int_{0}^{1} \int_{S^{d-1}} \xi g(\xi) \sigma(d \xi) r^{1-\alpha} d r=\int_{\{\|x\| \leq 1\}} x \nu^{\alpha}(d x),
$$

which proves (c) and concludes proof of the short-time part of the theorem.

Case (ii): Long time $(h \rightarrow \infty)$. Notice that under condition $[11), E\left\|X_{1}^{G T S}\right\|^{2}<\infty$ (see, e.g., [14, Theorem 25.3]). Thus, for each $h>0$,

$$
Z_{t}^{h}:=h^{-1 / 2}\left[X_{h t}^{G T S}-h t b_{h}\right]
$$

is a zero mean Lévy process with the cumulant of $Z_{1}^{h}$ given by

$$
\begin{aligned}
C_{Z_{1}^{h}}(y) & =h C_{\mu}\left(h^{-1 / 2} y\right)-i h^{1 / 2}\left\langle y, b_{h}\right\rangle \\
& =h \int_{\mathbb{R}^{d}}\left(e^{i\left\langle y, h^{-1 / 2} x\right\rangle}-1-i\left\langle y, h^{-1 / 2} x\right\rangle\right) \nu^{G T S}(d x) \\
& =\int_{\mathbb{R}^{d}}\left(e^{i\langle y, x\rangle}-1-i\langle y, x\rangle\right) \kappa^{h}(d x),
\end{aligned}
$$

where the measure $\kappa^{h}(B)=h \nu^{G T S}\left(h^{1 / 2} B\right), B \in \mathcal{B}\left(\mathbb{R}^{d}\right)$ is the Lévy measure of $Z_{1}^{h}$.

Similarly to the proof of the small time behavior, it is enough to verify the convergence in distribution at time $t=1$. Using [7, Theorem 15.14], the latter amounts to verifying the following three conditions: as $h \rightarrow \infty$,

(a') $\kappa^{h}$ converges vaguely to the null-measure on $\overline{\mathbb{R}^{d}} \backslash\{0\}$,

(b') $\int_{\{\|x\| \leq 1\}} x x^{\top} \kappa^{h}(d x) \rightarrow \Sigma$,

(c') $\int_{\{\|x\|>1\}} x \kappa^{h}(d x) \rightarrow 0$.

To prove (a') we take $\epsilon>0$. Then, as $h \rightarrow \infty$,

$$
\kappa^{h}(\{\|x\|>\epsilon\}) \leq \epsilon^{-2} \int_{\{\|x\|>\epsilon\}}\|x\|^{2} \kappa^{h}(d x)=\epsilon^{-2} \int_{\left\{\|x\|>\epsilon h^{1 / 2}\right\}}\|x\|^{2} \nu^{G T S}(d x) \rightarrow 0,
$$

which follows by 11). 
Now we consider (b'). Recall the definition of $\Sigma$ from 13 . Then, in view of (11),

$$
\int_{\{\|x\| \leq 1\}} x x^{\top} \kappa^{h}(d x)=\int_{\left\{\|x\| \leq h^{1 / 2}\right\}} x x^{\top} \nu^{G T S}(d x) \rightarrow \Sigma,
$$

as $h \rightarrow \infty$.

Finally, we consider $\left(c^{\prime}\right)$. We have

$$
\begin{aligned}
\left\|\int_{\{\|x\|>1\}} x \kappa^{h}(d x)\right\| & \leq \int_{\{\|x\|>1\}}\|x\| \kappa^{h}(d x) \leq \int_{\{\|x\|>1\}}\|x\|^{2} \kappa^{h}(d x) \\
& =\int_{\left\{\|x\|>h^{1 / 2}\right\}}\|x\|^{2} \nu^{G T S}(d x) \rightarrow 0,
\end{aligned}
$$

as $h \rightarrow \infty$. The proof is complete.

4. Absolute continuity with respect to a stable process. In this section, absolute continuity of GTS processes with respect to the associated stable process is studied. This should be compared with the results of [13, [6] and [3].

Recall that a process $\{X(t): t \geq 0\}$ in $\mathbb{R}^{d}$ is said to be canonical if $X(t, \omega)=\omega(t)$, $t \geq 0, \omega \in \Omega$, where $\Omega=D\left([0, \infty), \mathbb{R}^{d}\right) ; \Omega$ is equipped with the $\sigma$-algebra $\mathcal{F}=\sigma\{X(s)$ : $s \geq 0\}$ and the right-continuous natural filtration $\mathcal{F}_{t}=\bigcap_{s>t} \sigma\{X(u): u \leq s\}, t \geq 0$. The canonical process is completely described by a probability measure $P$ on $(\Omega, \mathcal{F})$. As usual, we set $\Delta X(t)=X(t)-X(t-)$. By $P_{\mid \mathcal{F}_{t}}$ we will denote the restriction of $P$ to the $\sigma$-algebra $\mathcal{F}_{t}$.

THEOREM 4.1. In the above setting consider two probability measures $P$ and $Q$ on $(\Omega, \mathcal{F})$ such that under $P$ the canonical process is a GTS process, $X_{t}=X_{t}^{G T S}$, with $\mathcal{L}\left(X_{1}^{G T S}\right)=$ $\mu$ determined by (7) and under $Q$ it is the canonical process is a $\alpha$-stable process, $X_{t}=$ $X_{t}^{\alpha}-t x_{0}$, with $\mathcal{L}\left(X_{1}^{\alpha}\right)=\mu^{\alpha}$ determined by $(9)$. Then, the following two statements are equivalent:

(1) $P_{\mid \mathcal{F}_{t}}$ and $Q_{\mid \mathcal{F}_{t}}$ are mutually absolutely continuous for every $t \in(0, \infty)$

(2) The following three conditions hold

(2.a) $\sigma$-almost everywhere on $S^{d-1}$

$$
\{\xi: g(\xi)=0\}=\left\{\xi: \int_{0}^{\infty} q(r, \xi) d r=0\right\}
$$

$$
\int_{S^{d-1}} \int_{0}^{1}\left(q(r, \xi)^{1 / 2}-g(\xi)^{1 / 2}\right)^{2} r^{-\alpha-1} d r \sigma(d \xi)<\infty
$$

$$
x_{0}= \begin{cases}0 & \text { if } \alpha \in(0,1), \\ \int_{S^{d-1}} \xi \int_{0}^{1}(q(r, \xi)-g(\xi)) r^{-\alpha} d r \sigma(d \xi) & \text { if } \alpha \in[1,2) .\end{cases}
$$

If $P_{\mid \mathcal{F}_{t}}$ and $Q_{\mid \mathcal{F}_{t}}$ are not mutually absolutely continuous for some $t>0$, then they are singular for all $t>0$.

Proof. In the proof we verify conditions for the equivalence of distributions of Lévy processes from [14, Theorem 33.1]. The first condition for (1) is that $\nu^{G T S}$ and $\nu^{\alpha}$ must 
be mutually absolutely continuous. We claim that this is equivalent to (2.a). Indeed, let $U=\left\{\xi \in S^{d-1}: g(\xi)=0\right\}$ and $U_{1}=\left\{\xi \in S^{d-1}: \int_{0}^{\infty} q(r, \xi) d r=0\right\}$. Then by (8)

$$
\nu^{\alpha}(\{r \xi: r>0, \xi \in U\})=0 .
$$

Consequently,

$$
0=\nu^{G T S}(\{r \xi: r>0, \xi \in U\})=\int_{U} \int_{0}^{\infty} r^{-\alpha-1} q(r, \xi) d r \sigma(d \xi),
$$

which shows $U \subset U_{1} \sigma$-almost everywhere. Proceeding in the reverse order yields $U_{1} \subset U$ $\sigma$-almost everywhere, which completes the proof of (2.a). Conversely, if $U=U_{1} \sigma$-almost everywhere, then for every $B \in \mathcal{B}\left(\mathbb{R}^{d}\right)$

$$
\begin{aligned}
\nu^{G T S}(B) & =\int_{U_{1}^{c}} \int_{0}^{\infty} 1_{B}(r \xi) q(r, \xi) r^{-\alpha-1} d r \sigma(d \xi) \\
& =\int_{U^{c}} \int_{0}^{\infty} 1_{B}(r \xi) q(r, \xi) r^{-\alpha-1} d r \sigma(d \xi) \\
& =\int_{S^{d-1}} \int_{0}^{\infty} 1_{B}(r \xi) \frac{q(r, \xi)}{g(\xi)} \mathbf{1}_{\{g(\xi)>0\}}(\xi) r^{-\alpha-1} d r g(\xi) \sigma(d \xi) \\
& =\int_{B} \frac{q(\|x\|, x /\|x\|)}{g(x /\|x\|)} \mathbf{1}_{\{g(x /\|x\|)>0\}}(x) \nu^{\alpha}(d x) .
\end{aligned}
$$

Thus $\nu^{G T S}$ is absolutely continuous with respect to $\nu^{\alpha}$, and since

$$
\nu^{G T S}(\{x: g(x /\|x\|)=0\})=\nu^{\alpha}(\{x: g(x /\|x\|)=0\})=0
$$

by (2.a), $\nu^{G T S}$ and $\nu^{\alpha}$ are equivalent with the Radon-Nikodym derivative

$$
\frac{d \nu^{G T S}}{d \nu^{\alpha}}(x)= \begin{cases}\frac{q(\|x\|, x /\|x\|)}{g(x /\|x\|)} & \text { if } g(x /\|x\|)>0 \\ 1 & \text { otherwise. }\end{cases}
$$

The second condition in [14, Theorem 33.1] for (1) is

$$
\int_{\mathbb{R}^{d}}\left(e^{\phi(x) / 2}-1\right)^{2} \nu^{\alpha}(d x)<\infty,
$$

where

$$
\phi(x):=\log \frac{d \nu^{G T S}}{d \nu^{\alpha}}(x) .
$$

Substituting (17) we get

$$
\begin{aligned}
\int_{\mathbb{R}^{d}}\left(e^{\phi(x) / 2}-1\right)^{2} \nu^{\alpha}(d x) & =\int_{\{g(\xi)>0\}} \int_{0}^{\infty}\left(\left(\frac{q(r, \xi)}{g(\xi)}\right)^{1 / 2}-1\right)^{2} r^{-\alpha-1} g(\xi) d r \sigma(d \xi) \\
& =\int_{S^{d-1}} \int_{0}^{\infty}\left(q(r, \xi)^{1 / 2}-g(\xi)^{1 / 2}\right)^{2} r^{-\alpha-1} d r \sigma(d \xi)
\end{aligned}
$$


where the last equality employs (2.a). Observe that

$$
\begin{aligned}
\int_{S^{d-1}} \int_{1}^{\infty} & \left(q(r, \xi)^{1 / 2}-g(\xi)^{1 / 2}\right)^{2} r^{-\alpha-1} d r \sigma(d \xi) \\
& \leq \int_{S^{d-1}} \int_{1}^{\infty} q(r, \xi) r^{-\alpha-1} d r \sigma(d \xi)+\int_{S^{d-1}} \int_{1}^{\infty} g(\xi) r^{-\alpha-1} d r \sigma(d \xi) \\
& =\nu^{G T S}(\{\|x\|>1\})+\nu^{\alpha}(\{\|x\|>1\})<\infty
\end{aligned}
$$

Thus,

$$
\int_{\mathbb{R}^{d}}\left(e^{\phi(x) / 2}-1\right)^{2} \nu^{\alpha}(d x)<\infty
$$

if and only if

$$
\int_{S^{d-1}} \int_{0}^{1}\left(q(r, \xi)^{1 / 2}-g(\xi)^{1 / 2}\right)^{2} r^{-\alpha-1} d r \sigma(d \xi)<\infty
$$

which is (2.b). The third condition in [14, Theorem 33.1] gives $x_{0}$ as in (2.c).

The last statement of the theorem comes from a general dichotomy result of [2].

REMARK 4.2. A direct application of Theorem 33.2 in [14 to the Radon-Nikodym derivative (17) yields the Radon-Nikodym derivative $\left.\frac{d P}{d Q}\right|_{\mathcal{F}_{t}}$ for each $t>0$.

REMARK 4.3. The critical condition in Theorem 4.1 for the absolute continuity is (2.b). It depends on the rate of convergence of $q(r, \xi)$ to $g(\xi)$ as $r \rightarrow 0$. We will state a (usually) simple to verify condition which yields (2.b). Suppose there exists a function $k(r)>0$ such that $\int_{0}^{1} k(r) r^{-1} d r<\infty$ such that

$$
\limsup _{r \rightarrow 0} \frac{1}{r^{\alpha} k(r)} \int_{S^{d-1}}\left(q(r, \xi)^{1 / 2}-g(\xi)^{1 / 2}\right)^{2} \sigma(d \xi)<\infty .
$$

Then condition (2.b) of Theorem 4.1 holds.

Proof. There exists $r_{0} \in(0,1]$ and a constant $M$ such that

$$
\int_{S^{d-1}}\left(q(r, \xi)^{1 / 2}-g(\xi)^{1 / 2}\right)^{2} \sigma(d \xi) \leq M k(r) r^{\alpha} \quad \forall r \in\left(0, r_{0}\right] .
$$

Hence

$$
\int_{S^{d-1}} \int_{0}^{r_{0}}\left(q(r, \xi)^{1 / 2}-g(\xi)^{1 / 2}\right)^{2} r^{-\alpha-1} d r \sigma(d \xi) \leq M \int_{0}^{r_{0}} k(r) r^{-1} d r<\infty .
$$

Then we estimate

$$
\begin{aligned}
\int_{S^{d-1}} \int_{r_{0}}^{1}\left(q(r, \xi)^{1 / 2}-g(\xi)^{1 / 2}\right)^{2} r^{-\alpha-1} d r \sigma(d \xi) \\
\leq \nu^{G T S}\left(\left\{\|x\|>r_{0}\right\}\right)+\nu^{\alpha}\left(\left\{\|x\|>r_{0}\right\}\right)<\infty
\end{aligned}
$$

similarly as in the proof of condition (2.b) of the above theorem. 
ExAmple 4.4 (A Tempered Stable Process). Let $q(r, \xi)=e^{-r f(\xi)}$, where $f: S^{d-1} \mapsto$ $(0, \infty)$. Here $g(\xi)=1$. Then,

$$
\begin{aligned}
\int_{S^{d-1}} & \left(q(r, \xi)^{1 / 2}-g(\xi)^{1 / 2}\right)^{2} \sigma(d \xi)=\int_{S^{d-1}}\left(e^{-r f(\xi) / 2}-1\right)^{2} \sigma(d \xi) \\
\leq & \frac{1}{4} r^{2} \int_{S^{d-1}} f(\xi)^{2} \sigma(d \xi) .
\end{aligned}
$$

Thus condition 18 holds with $k(r)=r^{2-\alpha}$, as long as $\int_{S^{d-1}} f(\xi)^{2} \sigma(d \xi)<\infty$.

EXAmple 4.5 (Lamperti Stable Processes). Let

$$
q(r, \xi)=\frac{e^{r f(\xi)} r^{\alpha+1}}{\left(e^{r}-1\right)^{\alpha+1}},
$$

where $f: S^{d-1} \rightarrow \mathbb{R}$ is such that $\beta:=\sup _{\xi \in S^{d-1}} f(\xi)<\alpha+1$. Here $g(\xi)=1$. Then, for $r \in(0,1]$

$$
\begin{aligned}
\int_{S^{d-1}} & \left(q(r, \xi)^{1 / 2}-g(\xi)^{1 / 2}\right)^{2} \sigma(d \xi)=\int_{S^{d-1}}\left(\frac{e^{r f(\xi) / 2} r^{(\alpha+1) / 2}}{\left(e^{r}-1\right)^{(\alpha+1) / 2}}-1\right)^{2} \sigma(d \xi) \\
& \leq 2 \frac{r^{\alpha+1}}{\left(e^{r}-1\right)^{\alpha+1}} \int_{S^{d-1}}\left(e^{r f(\xi) / 2}-1\right)^{2} \sigma(d \xi)+2\left(\frac{r^{(\alpha+1) / 2}}{\left(e^{r}-1\right)^{(\alpha+1) / 2}}-1\right)^{2} \sigma\left(S^{d-1}\right) \\
& \leq \frac{1}{2} C_{1} e^{\alpha+1} r^{2} \int_{S^{d-1}} f(\xi)^{2} \sigma(d \xi)+2 C_{2} r^{2} \sigma\left(S^{d-1}\right),
\end{aligned}
$$

where $C_{1}=\sup _{r \in(0,1]} \frac{r^{\alpha+1}}{\left(e^{r}-1\right)^{\alpha+1}}<\infty$ and $C_{2}=\sup _{r \in(0,1]} r^{-2}\left(\frac{r^{(\alpha+1) / 2}}{\left(e^{r}-1\right)^{(\alpha+1) / 2}}-1\right)^{2}<\infty$ by elementary calculus. Thus a Lamperti stable process is equivalent to an underlying stable process provided $\int_{S^{d-1}} f(\xi)^{2} \sigma(d \xi)<\infty$ (we take $k(r)=r^{2-\alpha}$ in (18). Such result was proved in [3, Theorem 5.1].

ExAmple 4.6 (A Not Equivalent Tempered Stable Process). In conclusion, we recall an example from [13. Example 4]. Consider $d=1$ for simplicity, $\sigma=\delta_{1}$ and $q(r)=q(r, 1)=$ $e^{-r^{\beta}}$, where $0<\beta \leq \frac{\alpha}{2}$. Since $1-e^{-x / 2} \geq x / 4$ when $x \in[0,1]$,

$$
\int_{0}^{1}\left(e^{-r^{\beta} / 2}-1\right)^{2} r^{-\alpha-1} d r \geq \frac{1}{16} \int_{0}^{1} r^{2 \beta-\alpha-1} d r=\infty .
$$

Thus (2.b) fails and the resulting tempered stable process is not equivalent to any stable process.

5. Series representation of GTS Lévy processes. In this section we examine two methods, LePage and Rejection Methods [12, to generate series representations of GTS Lévy processes. First, a series representation using LePage's Method is considered. It involves looking at a decomposition of the Lévy measure of the form:

$$
\nu^{G T S}(A)=\int_{S^{d-1}} \int_{0}^{\infty} 1_{A}(r \xi) \rho_{*}(d r, \xi) \sigma_{*}(d \xi)
$$

where $\sigma_{*}$ is a probability measure on $S^{d-1}$ and $\rho_{*}(\cdot, \xi)$ is a Lévy measure for $\sigma_{*}$-almost all $\xi \in S^{d-1}$. Such a decomposition is naturally provided by (1) if we take $\sigma_{*}(d \xi):=$ 
$\sigma(d \xi) / \sigma\left(S^{d-1}\right):=\sigma(d \xi) /\|\sigma\|$ and $\rho_{*}(\cdot, \xi)$ given by

$$
\rho_{*}([x, \infty), \xi)=\|\sigma\| \rho([x, \infty), \xi),
$$

where

$$
\rho([x, \infty), \xi):=\int_{x}^{\infty} q(r, \xi) r^{-\alpha-1} d r
$$

Since

$$
\int_{S^{d-1}} \int_{0}^{\infty}\left(1 \wedge r^{2}\right) \rho_{*}(d r, \xi) \sigma_{*}(d \xi)=\int_{\mathbb{R}^{d}}\left(1 \wedge\|x\|^{2}\right) \nu^{G T S}(d x)<\infty
$$

we get

$$
\int_{0}^{\infty}\left(1 \wedge r^{2}\right) \rho_{*}(d r, \xi)<\infty \quad \sigma_{*} \text {-almost everywhere on } S^{d-1},
$$

so that $\rho_{*}(\cdot, \xi)$ is a Lévy measure for $\sigma_{*}$-almost all $\xi \in S^{d-1}$. Define the right-continuous inverse of the tail function $x \mapsto \rho_{*}([x, \infty), \xi)$ as:

$$
\rho_{*}^{-1}(u, \xi):=\inf \left\{x>0: \rho_{*}([x, \infty), \xi)<u\right\} .
$$

Then

$$
\rho_{*}^{-1}(u, \xi)=\rho^{-1}(u /\|\sigma\|, \xi),
$$

where $\rho^{-1}(\cdot, \xi)$ is the right-continuous inverse of the function $x \mapsto \rho([x, \infty), \xi)$ given by 19 .

THEOREM 5.1. Let $\mu^{G T S}$ be a GTS distribution with the cumulant function (7), where $\nu^{G T S}$ is given by (1) and $b=0$. Put $\|\sigma\|:=\sigma\left(S^{d-1}\right)$ and let $T>0$ be fixed. Let $\left\{\Gamma_{i}\right\}_{i \geq 1}$ be a sequence of partial sums of iid standard exponential random variables. Let $\left\{U_{i}\right\}_{i \geq 1}$ be a sequence of iid uniform random variables on $[0, T]$ and let $\left\{V_{i}\right\}_{i \geq 1}$ be a sequence of random variables in $S^{d-1}$ with common distribution $\sigma(d \xi) /\|\sigma\|$. Assume that the sequences $\left\{\Gamma_{i}\right\}_{i \geq 1},\left\{U_{i}\right\}_{i \geq 1}$, and $\left\{V_{i}\right\}_{i \geq 1}$ are independent of each other. Then,

$$
X_{t}^{G T S}:=\sum_{i=1}^{\infty}\left\{\rho^{-1}\left(\frac{\Gamma_{i}}{\|\sigma\| T}, V_{i}\right) V_{i} \mathbf{1}_{\left\{U_{i} \leq t\right\}}-c_{i} \frac{t}{T}\right\}
$$

converges uniformly a.s. on $[0, T]$ to a Lévy process such that $\mathcal{L}\left(X_{1}^{G T S}\right)=\mu^{G T S}$, where $c_{i}=0$ when $\alpha \in(0,1)$ and for $\alpha \in[1,2)$

$$
c_{i}=\int_{i-1}^{i} E\left\{\rho^{-1}\left(\frac{s}{\|\sigma\| T}, V_{1}\right) V_{1} \mathbf{1}_{\left\{s \geq\|\sigma\| T \rho\left([1, \infty), V_{1}\right)\right\}}(s)\right\} d s .
$$

In particular, if the function $q$ does not depend on $\xi, q(r, \xi)=q(r)$, then $\rho^{-1}$ will not depend on $\xi$ either and the above series representation is simplified to the following:

$$
\sum_{i=1}^{\infty}\left\{\rho^{-1}\left(\frac{\Gamma_{i}}{\|\sigma\| T}\right) V_{i} \mathbf{1}_{\left\{U_{i} \leq t\right\}}-c_{i} \frac{t}{T}\right\}
$$

where $c_{i}=0$ when $\alpha \in(0,1)$ and for $\alpha \in[1,2)$

$$
c_{i}=E\left(V_{1}\right) \int_{i-1}^{i} \rho^{-1}\left(\frac{s}{\|\sigma\| T}\right) \mathbf{1}_{\{s \geq\|\sigma\| T \rho([1, \infty))\}}(s) d s .
$$

Proof. This representation is a special case of series representations considered in [12], where the pathwise uniform convergence of the series is proved in general. 
Practical applicability of series (20)-21) (e.g., for simulation of GTS processes) depends on whether or not $\rho^{-1}$ has a closed form. We will give a couple of examples where this is the case.

Example 5.2 (Lamperti Stable Processes). Recall that

$$
q(r, \xi)=\frac{e^{r f(\xi)} r^{\alpha+1}}{\left(e^{r}-1\right)^{\alpha+1}}
$$

Asssume that $f(\xi)=1$ for all $\xi \in S^{d-1}$. Then

$$
\rho([x, \infty), \xi)=\rho([x, \infty))=\int_{x}^{\infty} \frac{e^{r} r^{\alpha+1}}{\left(e^{r}-1\right)^{\alpha+1}} r^{-\alpha-1} d r=\alpha^{-1}\left(e^{x}-1\right)^{-\alpha},
$$

which implies that

$$
\rho^{-1}(u)=\ln \left(1+(\alpha u)^{-1 / \alpha}\right) .
$$

From Theorem 5.1 we get a series expansion of Lamperti stable processes on $[0, T]$ with $f(\xi):=1($ and $b=0$ in $(7))$

$$
X_{t}=\sum_{i=1}^{\infty}\left\{\ln \left(1+\left(\frac{\alpha \Gamma_{i}}{\|\sigma\| T}\right)^{-1 / \alpha}\right) V_{i} \mathbf{1}_{\left\{U_{i} \leq t\right\}}-c_{i} \frac{t}{T}\right\},
$$

where $c_{i}=0$ when $\alpha \in(0,1)$ and when $\alpha \in[1,2)$

$$
c_{i}=E\left(V_{1}\right) \int_{i-1}^{i} \log \left(1+\left(\frac{\alpha s}{\|\sigma\| T}\right)^{-1 / \alpha}\right) \mathbf{1}_{\{s \geq k\|\sigma\| T\}}(s) d s,
$$

where $k=\rho([1, \infty))=\alpha^{-1}(e-1)^{-\alpha}$. The representation $[22$ was also obtained in [3].

The next is the well-known series representation of stable processes.

Example 5.3 (Stable Processes). A stable process is a GTS process when we take $q(r, \xi) \equiv 1$. Then,

$$
\rho([x, \infty), \xi)=\rho([x, \infty))=\int_{x}^{\infty} r^{-\alpha-1} d r=\frac{x^{-\alpha}}{\alpha}
$$

which implies that

$$
\rho^{-1}(u)=(\alpha u)^{-\frac{1}{\alpha}}
$$

Thus,

$$
X_{t}=\sum_{i=1}^{\infty}\left\{\left(\frac{\alpha \Gamma_{i}}{\|\sigma\| T}\right)^{-\frac{1}{\alpha}} V_{i} \mathbf{1}_{\left\{U_{i} \leq t\right\}}-c_{i} \frac{t}{T}\right\}
$$

where $c_{i}=0$ when $\alpha \in(0,1)$ and for $\alpha \in[1,2)$

$$
c_{i}=E\left(V_{1}\right) \int_{i-1}^{i}\left(\left(\frac{\alpha s}{\|\sigma\| T}\right)^{-\frac{1}{\alpha}} \mathbf{1}_{\left\{s \geq \alpha^{-1}\|\sigma\| T\right\}}(s)\right) d s
$$

converges pathwise uniformly on $[0, T]$ to an $\alpha$-stable process with spectral measure $\sigma$ and $b=0$ in (7). 
EXAMPLE 5.4 (Exponentially tempered stable process). Consider a tempered stable process with $q(r, \xi)=e^{-r}$. Then,

$$
\rho([x, \infty), \xi)=\rho([x, \infty))=\int_{x}^{\infty} e^{-r} r^{-\alpha-1} d r
$$

does not have a closed form, neither does its inverse $\rho^{-1}(u)$.

The above example shows a simple case where the series representation based on the LePage method is difficult to apply in practice. For tempered stable processes a different kind series representations were introduced in [13. However, such representations will not work for arbitrary GTS processes. The next result develops the rejection method of Rosiński [12] for series representations of GTS processes which applies whenever the function $q$ is bounded and beyond.

THEOREM 5.5. Let $\mu^{G T S}$ be a GTS distribution with the cumulant function (7), where $\nu^{G T S}$ is given by 1 and $b=0$. Suppose there is a function $h:(0, \infty) \rightarrow \mathbb{R}_{+}$such that $\int_{0}^{\infty}\left(r^{2} \wedge 1\right) h(r) r^{-\alpha-1} d r<\infty$ and

$$
\sup _{\xi \in S^{d-1}} q(r, \xi) \leq h(r) \quad \text { for all } r>0 .
$$

Define

$$
\rho([x, \infty))=\int_{x}^{\infty} h(r) r^{-\alpha-1} d r
$$

and let $\rho^{-1}$ be the right-continuous inverse of the function $x \mapsto \rho([x, \infty))$. Put $\|\sigma\|:=$ $\sigma\left(S^{d-1}\right)$ and let $T>0$ be fixed.

Let $\left\{\Gamma_{i}\right\}_{i \geq 1}$ be a sequence of partial sums of iid standard exponential random variables. Let $\left\{U_{i}\right\}_{i \geq 1}$ be a sequence of iid uniform random variables on $[0, T]$ and let $\left\{V_{i}\right\}_{i \geq 1}$ be a sequence of random variables in $S^{d-1}$ with common distribution $\sigma(d \xi) /\|\sigma\|$. Let $\left\{W_{i}\right\}_{i \geq 1}$ be a sequence of iid uniform random variables on $[0,1]$. Assume that the sequences $\left\{\Gamma_{i}\right\}_{i \geq 1},\left\{U_{i}\right\}_{i \geq 1},\left\{V_{i}\right\}_{i \geq 1}$, and $\left\{W_{i}\right\}_{i \geq 1}$ are independent of each other. Define

$$
J_{i}:=\rho^{-1}\left(\frac{\Gamma_{i}}{\|\sigma\| T}\right), \quad i \geq 1
$$

and

$$
\beta_{i}:= \begin{cases}1 & \text { if } q\left(J_{i}, V_{i}\right) \geq h\left(J_{i}\right) W_{i} \\ 0 & \text { otherwise }\end{cases}
$$

Then,

$$
X_{t}^{G T S}:=\sum_{i=1}^{\infty}\left\{\beta_{i} J_{i} V_{i} \mathbf{1}_{\left\{U_{i} \leq t\right\}}-c_{i} \frac{t}{T}\right\}
$$

converges uniformly a.s. on $[0, T]$ to a Lévy process such that $\mathcal{L}\left(X_{1}^{G T S}\right)=\mu^{G T S}$, where $c_{i}=0$ when $\alpha \in(0,1)$ and for $\alpha \in[1,2)$

$$
c_{i}=E\left\{\mathbf{1}_{[i-1, i]}\left(\Gamma_{1}\right) e^{\Gamma_{1}} \beta_{1} J_{1} V_{1}\right\} .
$$

Proof. Consider a measure

$$
\lambda(B)=\int_{S^{d-1}} \int_{0}^{\infty} \mathbf{1}_{B}(r \xi) h(r) r^{-\alpha-1} d r \sigma(d \xi), \quad B \in \mathcal{B}\left(\mathbb{R}^{d}\right) .
$$


$\lambda$ is a Lévy measure by our assumption on integrability of $h$. Moreover, $\nu^{G T S}$ is absolutely continuous with respect to $\lambda$ and

$$
\frac{d \nu^{G T S}}{d \lambda}(x)=\frac{q(\|x\|, x /\|x\|)}{h(\|x\|)} \leq 1
$$

by (24). This is a case where the rejection method of [12] applies. $\left\{J_{i} V_{i}\right\}_{i \geq 1}$ is a Poisson point process in $\mathbb{R}^{d}$ with the intensity measure $\lambda$ obtained by LePage's method and the $\beta_{i}$ 's provide trimming of this process. The conclusion follows from direct applications of 12$]$.

REMARK 5.6. This theorem gives us a possibility to choose $\rho$ such that $\rho$ has a closed form. For example, if $h(r) \equiv 1$, then $\rho^{-1}(u)=(\alpha u)^{-1 / \alpha}$.

EXAmple 5.7 (Lamperti Stable Processes again). Consider a Lamperti stable process with

$$
q(r, \xi)=\frac{e^{r f(\xi)} r^{\alpha+1}}{\left(e^{r}-1\right)^{\alpha+1}},
$$

where $\gamma:=\sup _{\xi \in S^{d-1}} f(\xi)<\alpha+1$. In Example 5.2 we gave a series representation for the case $f(\xi) \equiv 1$. Surprisingly, the method of that example will mostly fail when $f(\xi) \equiv \gamma \neq 1$. This is because of the lack of a closed form for $q^{-1}(u)$.

In this example we will assume that $f(\xi) \leq 1, \xi \in S^{d-1}$. Then

$$
q(r, \xi)=\frac{e^{r f(\xi)} r^{\alpha+1}}{\left(e^{r}-1\right)^{\alpha+1}} \leq \frac{e^{r} r^{\alpha+1}}{\left(e^{r}-1\right)^{\alpha+1}}=: h(r) .
$$

Now we compute the quantities from Theorem 5.5 .

$$
\rho^{-1}(u)=\ln \left(1+(\alpha u)^{-1 / \alpha}\right),
$$

as in Example 5.2

$$
J_{i}:=\ln \left(1+\left(\frac{\alpha \Gamma_{i}}{\|\sigma\| T}\right)^{-1 / \alpha}\right)
$$

and

$$
\beta_{i}:= \begin{cases}1 & \text { if } e^{-J_{i}\left(1-f\left(V_{i}\right)\right)} \geq W_{i} \\ 0 & \text { otherwise }\end{cases}
$$

Then

$$
X_{t}:=\sum_{i=1}^{\infty}\left\{\beta_{i} J_{i} V_{i} \mathbf{1}_{\left\{U_{i} \leq t\right\}}-c_{i} \frac{t}{T}\right\}
$$

converges uniformly a.s. on $[0, T]$ to a Lamperti stable process (with $b=0$ in (7)), where $c_{i}=0$ when $\alpha \in(0,1)$ and for $\alpha \in[1,2)$

$$
c_{i}=E\left\{\mathbf{1}_{[i-1, i]}\left(\Gamma_{1}\right) e^{\Gamma_{1}} \beta_{1} J_{1} V_{1}\right\} .
$$

ExAmple 5.8 (Modified Tempered Stable Processes). Recall from Example 2.4 that

$$
q(r, \xi)= \begin{cases}2^{\frac{1-\alpha}{2}}\left(\Gamma\left(\frac{\alpha+1}{2}\right)\right)^{-1}\left(\lambda_{+} r\right)^{\frac{\alpha+1}{2}} K_{\frac{\alpha+1}{2}}\left(\lambda_{+} r\right) & \text { if } \xi=1, \\ 2^{\frac{1-\alpha}{2}}\left(\Gamma\left(\frac{\alpha+1}{2}\right)\right)^{-1}\left(\lambda_{-} r\right)^{\frac{\alpha+1}{2}} K_{\frac{\alpha+1}{2}}\left(\lambda_{-} r\right) & \text { if } \xi=-1,\end{cases}
$$


where $\lambda_{+}, \lambda_{-}>0, \alpha \in(0,2)$, and $K_{p}$ is the modified Bessel function of the second kind with an integral representation given by (5). Since $K_{p}(x)=K_{-p}(x)$, we get

$$
\begin{aligned}
K_{p}(x) & =K_{-p}(x)=\frac{1}{2}\left(\frac{x}{2}\right)^{-p} \int_{0}^{\infty} e^{-t-\frac{x^{2}}{4 t} t^{p-1} d t} \\
& \leq \frac{1}{2}\left(\frac{x}{2}\right)^{-p} \int_{0}^{\infty} e^{-t} t^{p-1} d t=\frac{\Gamma(p)}{2}\left(\frac{2}{x}\right)^{p} .
\end{aligned}
$$

Hence,

$$
x^{p} K_{p}(x) \leq 2^{p-1} \Gamma(p), \quad x>0 .
$$

Using this bound with $p=(\alpha+1) / 2$ we get that

$$
\sup _{\xi \in S^{d-1}} q(r, \xi) \leq 1 \quad \text { for all } r>0 .
$$

Therefore, we use the rejection method with

$$
h(r) \equiv 1 .
$$

Now we compute the quantities from Theorem 5.5

$$
\begin{gathered}
\rho(u)=(\alpha u)^{-1 / \alpha}, \\
J_{i}:=\left(\frac{\alpha \Gamma_{i}}{\|\sigma\| T}\right)^{-1 / \alpha},
\end{gathered}
$$

and

$$
\beta_{i}:= \begin{cases}1 & \text { if } q\left(J_{i}, V_{i}\right) \geq W_{i} \\ 0 & \text { otherwise }\end{cases}
$$

Then,

$$
X_{t}:=\sum_{i=1}^{\infty}\left\{\beta_{i} J_{i} V_{i} \mathbf{1}_{\left\{U_{i} \leq t\right\}}-c_{i} \frac{t}{T}\right\}
$$

converges uniformly a.s. on $[0, T]$ to a MTS Lévy process (with $b=0$ in $(7)$ ), where $c_{i}=0$ when $\alpha \in(0,1)$ and for $\alpha \in[1,2)$

$$
c_{i}=E\left\{\mathbf{1}_{[i-1, i]}\left(\Gamma_{1}\right) e^{\Gamma_{1}} \beta_{1} J_{1} V_{1}\right\} .
$$

EXAmple 5.9 (Tempered Stable Processes). Special series representations for proper tempered stable processes were constructed in [13. They are based on intrinsic characteristics of such processes. Here we will only indicate that the rejection method of Theorem 5.5 can be applied as well to obtain alternative representations. Indeed, since

$$
\sup _{\xi \in S^{d-1}} q(r, \xi) \leq 1, \quad r>0
$$

we may take $h(r) \equiv 1$ in Theorem 5.5 to obtain series representations based on trimming of jumps of the associated stable processes.

\section{References}

[1] O. E. Barndorff-Nielsen and N. Shephard, Non-Gaussian Ornstein-Uhlenbeck-based models and some of their uses in financial economics, J. Roy. Statist. Soc. Ser. B 63 (2001), 1-42. 
[2] P. L. Brockett and H. G. Tucker, A conditional dichotomy theorem for stochastic processes with independent increments, J. Multivariate Anal. 7 (1977), 13-27.

[3] M. E. Caballero, J. C. Pardo, and J. L. Pérez, On Lamperti stable processes, Probability and Mathematical Statistics 30 (2010), 1-28.

[4] P. Carr, H. Geman, D. B. Madan and M. Yor, The fine structure of asset returns: An empirical investigation, J. Business 75 (2002) 303-325.

[5] S. Cohen and J. Rosiński, Gaussian approximation of multivariate Lévy processes with applications to simulation of tempered stable processes, Bernoulli 13 (2007) 195-210.

[6] C. Houdré and R. Kawai, On layered stable processes, Bernoulli 13 (2007) 252-278.

[7] O. Kallenberg, Foundations of Modern Probability, 2nd ed., Springer-Verlag, 2002.

[8] Y. S. Kim, S. T. Rachev, B. M. Chung, and M. L. Bianchi, The modified tempered stable distribution, Garch models, and option pricing, Probability and Mathematical Statistics 29 (2009), 91-117.

[9] I. Koponen, Analytic approach to the problem of convergence of truncated Lévy flights towards the Gaussian stochastic process, Phys. Rev. E 52 (1995), 1197-1199.

[10] R. N. Mantegna and H. E. Stanley, Stochastic process with ultraslow convergence to a Gaussian: The truncated Lévy flight, Phys. Rev. Lett. 73 (1994), 2946-2949.

[11] E. A. Novikov, Infinitely divisible distributions in turbulence, Phys. Rev. E 50 (1994), R3303-R3305.

[12] J. Rosiński, Series representations of Lévy processes from the perspective of point processes, in: Lévy Processes - Theory and Applications, and Analysis (Edmonton, 1973), O. E. Barndorff-Nielsen, T. Mikosch, S. I. Resnick (eds.), Birkhäuser, Boston, 2001, 401-415.

[13] J. Rosiński, Tempering stable processes, Stochastic Processes and their Applications 117 (2007), 677-707.

[14] K. Sato, Lévy Processes and Infinitely Divisible Distributions, Cambridge University Press, 1999.

[15] G. Terdik and W. A. Woyczyński, Rosiński measures for tempered stable and related Ornstein-Uhlenbeck processes, Probability and Mathematical Statistics 26 (2006), 213-243. 